\title{
Analysis of the Desulfovibrio gigas transcriptional unit containing rubredoxin (rd) and rubredoxin-oxygen oxidoreductase (roo) genes and upstream ORFs
}

\section{Servicos $9^{\circ} \circ$ $\mathrm{NCB}$}

\author{
Print E-mail Add to Marked List Save to EndNote Web \\ Save to EndNote, RefMan, ProCite more options
}

Author(s): Silva G, Oliveira S, LeGall J, Xavier AV, Rodrigues-Pousada C

Source: BIOCHEMICAL AND BIOPHYSICAL RESEARCH COMMUNICATIONS Volume: 280 Issue: 2 Pages: 491-502 Published: JAN 192001

Times Cited: 19 References: 42 ExGitation Map

Abstract: Rubredoxin-oxygen oxidoreductase, an $86-\mathrm{kDa}$ homodimeric flavoprotein, is the final component of a soluble electron transfer chain that couples NADH oxidation with oxygen reduction to water from the sulfate-reducing bacterium Desulfovibrio gigas. A 4.2-kb fragment Of $\mathrm{D}$. gigas chromosomal DNA containing the roo gene and the rubredoxin gene was sequenced, Additional open reading frames designated as ORF-1, ORF-2, and ORF-3 were also identified in this DNA fragment. ORF-1 encodes a protein exhibiting homology to several proteins of the short-chain dehydrogenase/reductase family of enzymes. The N-terminal coenzyme-binding pattern and the active-site pattern characteristic of short chain dehydrogenase/reductase proteins are conserved in ORF-1 product. OFF-2 does not show any significant homology with any known protein, whereas ORF-3 encodes a protein having significant homologies with the branched-chain amino acid transporter AzIC protein family. Northern blot hybridization analysis with rd and roo-specific probes identified a common 1.5-kb transcript, indicating that these two genes are cotranscribed. The transcription start site was identified by primer extension analysis to be a guanidine 87 bp upstream the ATG; start codon of rubredoxin, The transcript size indicates that the rd-roo mRNA terminates downstream the roo-coding unit. Putative -10 and -35 regulator regions of a sigma (70)-type promoter, having similarity with E. coli sigma (70) promoter elements, are found upstream the transcription start site. Rubredoxin-oxygen oxidoreductase and rubredoxin genes are shown to be constitutively and abundantly expressed. Using the data available from different prokaryotic genomes, the rubredoxin genomic organization and the first tentative to understand the phylogenetic relationships among the flavoprotein family are reported in this study. (C) 2001 Academic Press.

Document Type: Article

Language: English

Author Keywords: Desulfovibrio gigas; rubredoxin-oxygen oxidoreductase; flavoproteins

KeyWords Plus: SUPEROXIDE-DISMUTASE; VULGARIS HILDENBOROUGH; RHODOBACTERCAPSULATUS; ALCOHOL-DEHYDROGENASE; ELECTRON-TRANSPORT; REDUCING BACTERIA; NITROGEN-FIXATION; ESCHERICHIA-COLI; SULFATE; ANAEROBE

Reprint Address: Silva, G (reprint author), Univ Nova Lisboa, Inst tecnol Quim \& Biol, Apartado 127, P2781901 Oeiras, Portugal

Addresses:

1. Univ Nova Lisboa, Inst tecnol Quim \& Biol, P-2781901 Oeiras, Portugal

2. Univ Evora, Dept Biol, Evora, Portugal

3. Univ Georgia, Dept Biochem \& Mol Biol, Athens, GA 30602 USA 